\title{
Fragmentation of Millimeter-Size Hypervelocity Projectiles on Combined Mesh-Plate Bumpers
}

\author{
Aleksandr Cherniaev and Igor Telichev \\ Department of Mechanical Engineering, University of Manitoba, E2-327 EITC, 75A Chancellors Circle, Winnipeg, \\ $M B$, Canada R3T 5 V6 \\ Correspondence should be addressed to Aleksandr Cherniaev; aleksandr.cherniaev@umanitoba.ca
}

Received 29 March 2017; Accepted 12 July 2017; Published 16 August 2017

Academic Editor: Laszlo Toth

Copyright ( 2017 Aleksandr Cherniaev and Igor Telichev. This is an open access article distributed under the Creative Commons Attribution License, which permits unrestricted use, distribution, and reproduction in any medium, provided the original work is properly cited.

\begin{abstract}
This numerical study evaluates the concept of a combined mesh-plate bumper as a shielding system protecting unmanned spacecraft from small $(1 \mathrm{~mm})$ orbital debris impacts. Two-component bumpers consisting of an external layer of woven mesh (aluminum or steel) directly applied to a surface of the aluminum plate are considered. Results of numerical modeling with a projectile velocity of $7 \mathrm{~km} / \mathrm{s}$ indicate that, in comparison to the steel mesh-combined bumper, the combination of aluminum mesh and aluminum plate provides better fragmentation of small hypervelocity projectiles. At the same time, none of the combined mesh/plate bumpers provide a significant increase of ballistic properties as compared to an aluminum plate bumper. This indicates that the positive results reported in the literature for bumpers with metallic meshes and large projectiles are not scalable down to millimeter-sized particles. Based on this investigation's results, a possible modification of the combined mesh/plate bumper is proposed for the future study.
\end{abstract}

\section{Introduction}

Orbital debris impacts represent a serious threat to the operational integrity of space vehicles. Unlike manned spacecraft that are conservatively designed to be protected against lowprobability impacts of centimeter-size and even larger orbital debris (crew safety reasons), unmanned satellites are typically being designed with lower margins of safety against more probable impacts of small, millimeter-sized particles [1]. It should be noted that because of the extremely high velocities of orbital debris (over $7 \mathrm{~km} / \mathrm{s}$ ), even $1 \mathrm{~mm}$-sized objects can cause significant damage to spacecraft onboard systems. Such systems, therefore, must be adequately protected.

Orbital debris protection is typically represented by a "bumper" placed at some standoff from an onboard system requiring protection [2]. The bumper's function is to break the projectile into a cloud of fragments (also known as "debris cloud") that expands while moving across the standoff. The expansion results in distributing the initial impactor energy and momentum over a wide area on the shielded component ("rear wall") and, thus, reduces the severity of the damage. This principle is illustrated in Figure 1.

Inspired by the need to design protection for habitable modules of the International Space Station, most of the up-todate investigations consider hypervelocity impacts of large, centimeter-size objects on different kinds of bumpers and protective layers (e.g., $[3,4])$. Contrarily, only a few studies exist that deal with protecting against small orbital debris [1]. This study investigates fragmentation of small hypervelocity projectiles on combined mesh-plate (discrete-continuous) bumpers.

In its "conventional" design, the protective bumper is monolithic and made of an isotropic material, typically aluminum, known for its ability to efficiently break up mediumdensity projectiles. The aluminum monolithic bumper is considered as a "reference configuration" throughout this study. Other possible designs of the shielding systems are continuously being sought with the ultimate purpose of increasing their structural weight efficiency. In this regard, applying metallic meshes was found to be beneficial because 


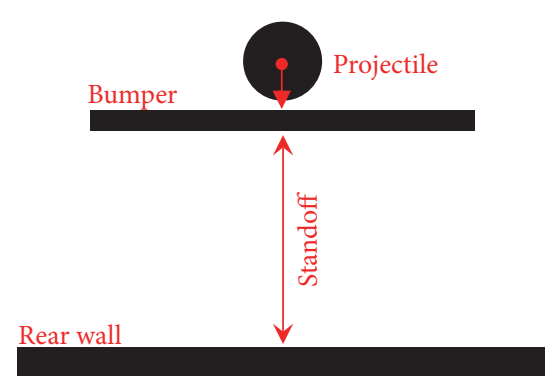

(a)

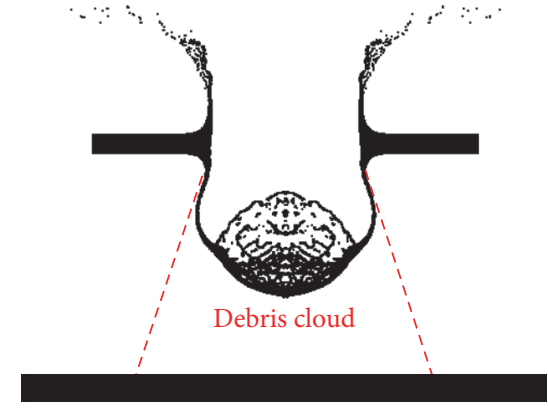

(b)

FIGURE 1: Schematic of an orbital debris shield before impact (a) and after perforation of the bumper (b).

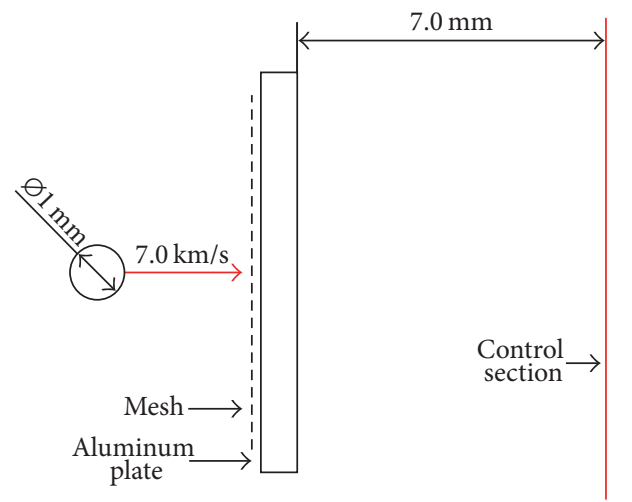

FIGURE 2: Initial setup for numerical experiments on combined mesh/plate bumpers.

of their low areal density. Fragmentation of centimeter-size hypervelocity projectiles on stand-alone metallic meshes was experimentally studied in $[5,6]$. The meshes demonstrated the ability to significantly decelerate the projectiles [5] and breaking them up by forming jets of material that "squeeze" through the mesh apertures [6].

Experimental studies conducted by NASA $[7,8]$ showed that so-called mesh double-bumper shield, consisting of spaced discrete (mesh), continuous (aluminum plates), and flexible (fabrics) layers, may have lower ballistic weight compared to the conventional shield with a continuous bumper. It should be noted, however, that shielding systems with multiple spaced layers typically require higher nonballistic parasitic weight for fasteners, spacers, and so forth [4]. In addition, use of multiwall shielding systems for protection against millimeter-sized objects may be impractical, as it may result in very low thickness of individual layers, which can easily buckle and permanently deform during satellite launching.

In the present study, we investigate the ballistic performance of combined mesh/plate bumper with an external layer of fine woven mesh directly applied on a surface of an aluminum plate and supported on it (Figure 2). Considering ballistic weight, mesh-plate bumpers may be advantageous, as they either increase the total thickness of a bumper (in case of aluminum mesh) while maintaining the same areal density as a monolithic aluminum bumper or facilitate improved initial breakup of projectiles because of their interaction with materials of higher shock impedance than aluminum (in the case of using steel mesh).

All investigations in this study were conducted by means of numerical modeling using SPH solver of ANSYS AUTODYN hydrocode [9] for a $1 \mathrm{~mm}$ aluminum projectile impacting the shields at a speed of $7 \mathrm{~km} / \mathrm{s}$.

\section{Numerical Modeling}

2.1. Modeled Bumper Configurations. Schematic representation of the layout of numerical experiments is shown in Figure 2. Here, the combined mesh/plate bumper consists of a layer of metallic mesh (aluminum or steel) backed by an aluminum plate. First, the discrete layer (mesh) breaks the projectile, which is followed by propagation of the formed smaller fragments (size smaller than the mesh's aperture) through the continuous medium (e.g., aluminum plate) resulting in their further disruption and deceleration. To compare the parameters of the fragment clouds, a "control section" was defined at a distance of $7 \mathrm{~mm}$ from the bumper (Figure 2).

Weight and areal density of all mesh/plate bumper configurations were equivalent to those of a $1 \mathrm{~mm}$ thick aluminum plate bumper. The latter was considered as a reference configuration for comparison.

Two types of meshes have been considered in this study: mesh made of Al6061-T6 aluminum alloy and steel mesh. Steel mesh is typically made of stainless steel. However, because of a lack of the full set of material constants specific to stainless steel, 4340 steel properties have been used for modeling steel mesh assuming an adequate level of similarity in the behavior of these materials under shock loading. A $1 \mathrm{~mm}$ diameter projectile was modeled as it was made of unalloyed aluminum Al1100-H12 and had a velocity of $7 \mathrm{~km} / \mathrm{s}$. Material models used to represent the projectiles, meshes, and plates' behavior under hypervelocity impact conditions are described in Section 2.2.

Mesh efficiency as a bumper depends on the mesh's geometrical parameters [4], which are the wire diameter 


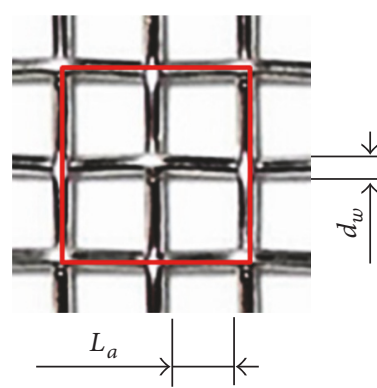

(a)

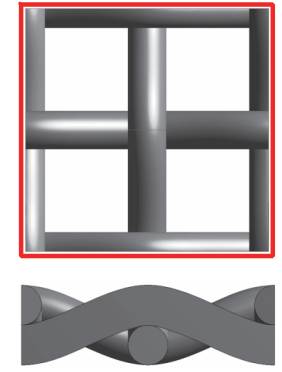

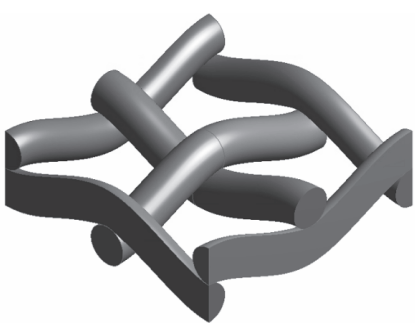

(b)

FIGURE 3: Geometric modeling of a woven metallic mesh: (a) metallic mesh; (b) geometric model of a unit cell.

$d_{w}$ and the size of the aperture $L_{a}$ (see Figure 3(a)). From this perspective, the simplest choice would be to use mesh with the smallest possible aperture. However, a typical mesh of submillimeter aperture along with the reduction of $L_{a}$ exhibits

(a) reduced wire diameter, which reduces mesh efficiency as an impact protective element;

(b) reduced aperture-to-wire diameter ratio $\left(L_{a} / d_{w}\right)$ and, consequently, increased areal density. This, in turn, reduces the advantage of a mesh over a continuous material bumper.

On the other hand, using coarse meshes with $L_{a} \geq d_{p}$ (projectile diameter) would not be beneficial, as projectiles may get through the mesh aperture without being damaged. With these considerations in mind, a mesh with the following geometrical parameters has been chosen: aperture size $L_{a}=$ $0.39 \mathrm{~mm}$ and wire diameter $d_{w}=0.18 \mathrm{~mm}$, which makes the projectile diameter-to-aperture size ratio $d_{p} / L_{a}=2.56$ and the aperture-to-wire diameter ratio $L_{a} / d_{w}=2.17$.

As a result of the three-dimensional structure of the woven mesh, the mesoscale approach to the representation of its geometry has been utilized. The geometrical model of a unit cell (translation unit) of the metallic mesh is shown in Figure 3(b), which displays that the mesoscale approach enables realistic representation of the winding pattern of a mesh in numerical modeling.

As aforementioned, another element of a combined bumper configuration is an aluminum plate. To obtain comparable results, the weight and areal density of all combined bumpers were equivalent to those of a $1 \mathrm{~mm}$ thick aluminum plate bumper. This was achieved by varying the thicknesses of the aluminum plates, whereas the mesh parameters (wire diameter and aperture size) were the same for both aluminum and steel meshes. The corresponding thicknesses of the aluminum plates in the mesh/plate bumper layouts are given in Table 1. It can be noticed that using a steel mesh significantly reduces the thickness of the aluminum plate of the combined bumper because of the steel's high density.

The SPH method (smooth particles hydrodynamics) has been used for all simulations performed in this study. It is a mesh-free Lagrangian method that uses "particles" representing continua as interpolation points to estimate values
TABLE 1: Thicknesses of aluminum plates for different bumper configurations.

\begin{tabular}{lc}
\hline Type of bumper & Thickness of aluminum plate \\
\hline All-aluminum (no mesh) & $1.000 \mathrm{~mm}$ \\
Al mesh + Al plate & $0.905 \mathrm{~mm}$ \\
Steel mesh + Al plate & $0.723 \mathrm{~mm}$ \\
\hline
\end{tabular}

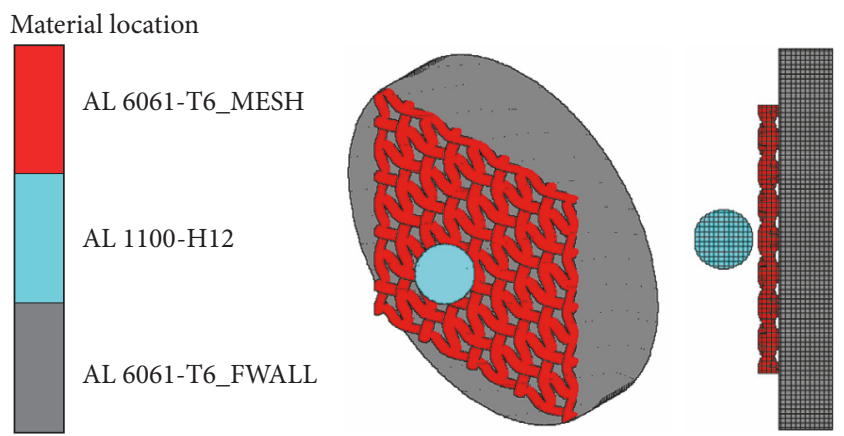

FIGURE 4: SPH model with mesoscale representation of a mesh bumper.

of functions and their spatial derivatives [10]. Advantages of the SPH over mesh-based methods in simulations of hypervelocity impacts are the absence of the mesh tangling problems and the lack of the need to use the artificial erosion mechanism. SPH particles "naturally" form discontinuities in the continuum such as cracking, penetration, and fragmentation as particles are forced to separate during the penetration event. The method has been validated by comparison to experimental data for a range of hypervelocity impact problems $[11,12]$.

As a result of the symmetry of the problem, only a quarter of each configuration has been modeled whereas the rest has been represented using corresponding symmetry constraints. This technique allows a significant reduction of the computational time. The SPH model of a combined meshplate bumper and a projectile is shown in Figure 4 (symmetric parts are shown using "mirror reflection" of the quartermodel). The main parameter of SPH particles is a smoothing 


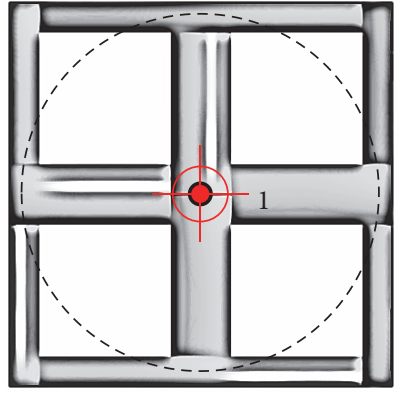

(a)

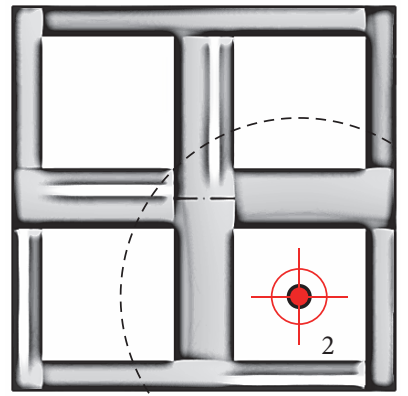

(b)

FIGURE 5: Positioning of the center of impact in numerical experiments: (a) projectile aligned with crossover point; (b) projectile aligned with the center of aperture.

length, which to some extent is equivalent to the element size in finite element calculations. In this study, the smoothing length was constant throughout the simulations and equal to $0.02 \mathrm{~mm}$. This resulted in 50 particles along the projectile diameter and approximately 16,400 particles representing the quarter of a $1 \mathrm{~mm}$ projectile. The number of SPH particles used to represent mesh/plate bumpers varied depending on configuration (steel or aluminum mesh), but in all cases was over one million. It should be noted that additional definition of contact between mesh and plate was not needed, as they were both represented by SPH particles: in SPH method particles' interaction logic is solely based on the smoothing length definition.

As a result of the irregular three-dimensional structure of the metallic mesh, the impact of a projectile at different locations may have different consequences in terms of damage to the shielded component. In this study, we considered two cases: impact of a projectile at the crossover point (wires' intersection; see Figure 5(a)) and impact of a projectile in the center of aperture (Figure 5(b)). The former case was considered as the most optimistic, as the wires' intersection provided a significant increase in the overall bumper thickness. The latter case was considered as the worst scenario. It is believed that all other cases are somewhere between those two in terms of damage to the spacecraft system that is placed behind the bumper.

2.2. Material Models. Material models for hypervelocity impact simulations typically consist of three general parts: an equation of state, a strength model, and a failure model. The equation of state represents the relationship between the hydrostatic pressure, the local density, and the local specific energy; strength model is required to relate deviatoric stresses and strains; and failure model is used for simulating failure of materials in terms of their inability to withstand tensile stresses exceeding its local tensile strength. According to this, the following subsections consider individually each part of the material models used in this study.

2.2.1. Equation of State. Mie-Gruneisen equation of state [13] has been used for all materials considered in this study. It establishes relationship between pressure, density, and specific energy of a solid material subjected to shock loading in the following form:

$$
p=p_{H}+\Gamma \rho \cdot\left(e-e_{H}\right)
$$

where $p, e$, and $\rho$ are pressure, specific energy, and density of the material correspondingly; $\Gamma$ is Gruneisen gamma (constant); $p_{H}$ and $e_{H}$ are the reference pressure and specific energy determined from material's shock Hugoniot. In turn, to define the shock Hugoniot, linear relationship between shock velocity $\left(U_{s}\right)$ and particle velocity $\left(U_{p}\right)$ was used:

$$
U_{s}=C_{0}+S \cdot U_{p},
$$

where $C_{0}$ is the bulk acoustic sound speed and $S$ is the slope of the $U_{s}-U_{p}$ relationship. Given $C_{0}$ and $S$ provided as material properties and using conservation of mass, momentum, and energy equations (e.g., in the form of the RankineHugoniot conservation equations [14]), $p_{H}$ and $e_{H}$ in (1) can be represented as functions of material density:

$$
p_{H}=\frac{\rho_{0} C_{0}^{2} \mu(1+\mu)}{[1-(S-1) \mu]^{2}},
$$

where $\mu=\left(\rho / \rho_{0}\right)-1, \rho_{0}$ is the initial density, and

$$
e_{H}=\frac{1}{2}\left(\frac{p_{H}}{\rho_{0}}\right)\left(\frac{\mu}{1+\mu}\right) .
$$

2.2.2. Strength Model. Although equation of state effectively describes the behavior of materials subjected to shock loading in the course of hypervelocity impact, strength effects may also be important or even dominating in so-called "far-field" and "late-time" regimes. Strength behavior of all materials considered in this study has been represented in terms of Johnson-Cook model [15], which is a typical choice for metals subjected to high strains, high strain rates, and high temperatures.

Application of Johnson-Cook strength and failure models to hypervelocity impact problems has been studied in $[1,16]$. In [16] it was found that the models are applicable for accurate predictions of target hole diameters from hypervelocity 
TABLE 2: Properties of materials.

\begin{tabular}{|c|c|c|c|c|c|}
\hline Component of material model & Property & Dimensionality & Al1100-H12 & Al6061-T6 & 4340 Steel \\
\hline \multirow{6}{*}{ Equation of state } & Reference density & $\mathrm{g} / \mathrm{cm}^{3}$ & 2.707 & 2.703 & 7.860 \\
\hline & Gruneisen gamma & - & 1.970 & 1.970 & 1.670 \\
\hline & $C_{0}$ & $\mathrm{~m} / \mathrm{s}$ & 5386 & 5240 & 4578 \\
\hline & $S$ & & 1.339 & 1.400 & 1.330 \\
\hline & Ref. temperature & $\mathrm{K}$ & 293 & 293 & 293 \\
\hline & Specific heat & $\mathrm{J} / \mathrm{kgK}$ & 884 & 885 & 477 \\
\hline \multirow{8}{*}{ Strength model } & Shear modulus & $\mathrm{MPa}$ & 24722 & 26000 & 77000 \\
\hline & Yield stress & $\mathrm{MPa}$ & 148 & 324 & 792 \\
\hline & Hardening constant & $\mathrm{MPa}$ & 345 & 114 & 510 \\
\hline & Hardening exponent & - & 0.183 & 0.420 & 0.260 \\
\hline & Strain rate constant & - & 0.001 & 0.002 & 0.014 \\
\hline & Thermal softening exponent & - & 0.859 & 1.340 & 1.030 \\
\hline & Melting temperature & K & 893 & 925 & 1793 \\
\hline & Reference strain rate & $/ \mathrm{s}$ & 1.000 & 1.000 & 1.000 \\
\hline \multirow{5}{*}{ Failure model } & Damage constant, $D_{1}$ & - & 0.071 & -0.770 & 0.050 \\
\hline & Damage constant, $D_{2}$ & - & 1.248 & 1.450 & 3.440 \\
\hline & Damage constant, $D_{3}$ & - & -1.142 & -0.470 & -2.120 \\
\hline & Damage constant, $D_{4}$ & - & 0.147 & 0.000 & 0.002 \\
\hline & Damage constant, $D_{5}$ & - & 1.000 & 1.600 & 0.610 \\
\hline
\end{tabular}

impacts onto thin aluminum targets. In [1], Johnson-Cook strength and failure models were found to be suitable for adequate predictions of Whipple shield's bumper hole diameter, rear wall damage, and bulging resulted from hypervelocity impacts.

In Johnson-Cook model, the yield stress of material is represented as

$$
Y=\left[A+B \cdot \varepsilon_{p}^{n}\right] \cdot\left[1+C \cdot \ln \dot{\varepsilon}_{p}^{*}\right] \cdot\left[1-T_{H}^{m}\right]
$$

where $\varepsilon_{p}, \dot{\varepsilon}_{p}{ }^{*}$, and $T_{H}$ are an effective plastic strain, normalized effective plastic strain rate, and homologous temperature $\left(T_{H}=\left(T-T_{\text {room }}\right) /\left(T_{\text {melt }}-T_{\text {room }}\right)\right)$, correspondingly; $A$, $B, C, n$, and $m$ are material constants, namely, initial yield strength, hardening constant, strain rate constant, hardening exponent, and thermal softening exponent. In Johnson-Cook plasticity model, calculation of plastic strain is based on the von Mises yield surface and the associated flow rule. Expression in the first brackets of (5) gives the yield stress as a function of plastic strain; the second set of brackets represents the effects of strain rate on stress; and the third set of brackets determines thermal softening of a material. It should be noted that as the temperature reaches melting temperature of the material $\left(T_{\text {melt }}\right)$, its yield strength drops to zero. As at high strain rates the deformation process is mainly adiabatic, the temperature in (5) is calculated by means of converting $90 \%$ of plastic work into heat.

2.2.3. Failure Model. In this study, Johnson-Cook failure model [15] has been used for all materials. This model defines dynamic fracture strain as a function of pressure, strain rate, and temperature in the following manner:

$$
\begin{aligned}
\varepsilon^{f}= & {\left[D_{1}+D_{2} \cdot \exp \left(D_{3} \cdot \sigma^{*}\right)\right] \cdot\left[1+D_{4} \cdot \ln \left|\dot{\varepsilon}_{p}^{*}\right|\right] } \\
& \cdot\left[1+D_{5} \cdot T_{H}\right],
\end{aligned}
$$

where $\sigma^{*}$ and $\dot{\varepsilon}_{p}{ }^{*}$ are dimensionless pressure-stress ratio and dimensionless strain rate, correspondingly; $D_{i}$ are damage parameters of the material. The dimensionless pressure-stress ratio is defined as $\sigma^{*}=\sigma_{m} / \bar{\sigma}$, where $\sigma_{m}$ is the average of the three normal stresses and $\bar{\sigma}$ is the von Mises equivalent stress. Damage is then defined as the ratio of the incremental effective plastic strain in an integration cycle and equivalent strain to fracture under the current conditions of strain rate, temperature, pressure, and equivalent stress: $D=\sum\left(\Delta \varepsilon / \varepsilon^{f}\right)$. Fracture of material occurs instantaneously when $D=1.0$.

2.2.4. Properties of Materials. Properties of all materials, in terms of the material model described above, were employed from the literature and based on experimental data (see Table 2). The following sources of information were used: [16] for the properties of Al6061-T6, where the material model was successfully used for hypervelocity impact simulations; [17] for Al1100-H12, where the material model was used to simulate ballistic impacts; and [15] for 4340 steel.

\section{Results and Discussion}

3.1. Fragmentation Model Verification. To ensure that the combination of the SPH method, the particular discretization scheme, and the material models used in this study are 


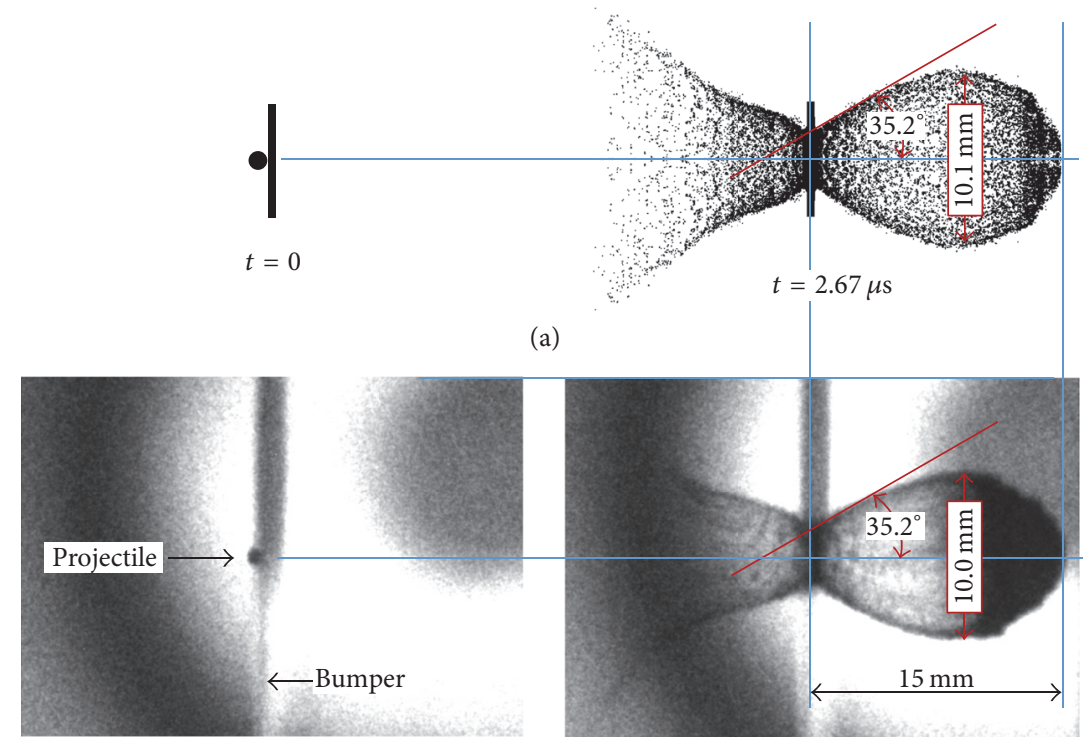

(b)

FIGURE 6: Results of numerical modeling (a) and experimental observations (b).

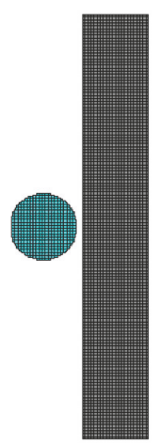

(a)

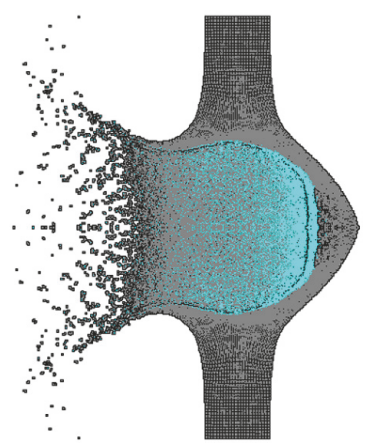

(b)

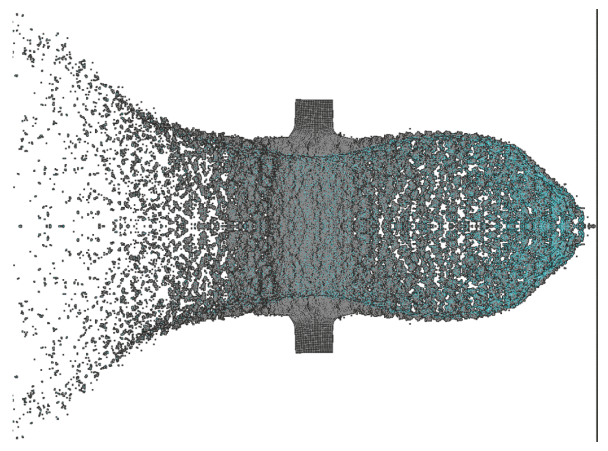

(c)

FIGURE 7: Impact of an aluminum projectile on an aluminum bumper: (a) $t=0$; (b) $t=0.55 \mu \mathrm{s}$; (c) $t=2.20 \mu \mathrm{s}$.

suitable for the adequate modeling of hypervelocity fragmentation, a hypervelocity impact experiment was conducted and then compared with the results of the numerical modeling. In the experiment, a $1 \mathrm{~mm}$ aluminum projectile $(0.047 \mathrm{~g})$ was accelerated by a two-stage light gas gun to the speed of $6.847 \mathrm{~km} / \mathrm{s}$ to hit a $0.8 \mathrm{~mm}$ thick aluminum bumper (Al6061T6).

The shape the fragment cloud formed on the bumper's perforation was captured using a high-speed camera, $2.67 \mu \mathrm{s}$ after impact. The photograph, as well as the results of numerical modeling obtained for the same conditions, is illustrated in Figure 6. Comparison of the overall shape of the fragment clouds presented in Figure 6, their lengths, cone angles, and diameters, as well as the shapes of ejecta clouds (backward-moving fragments), indicate that the employed numerical scheme is capable of accurately representing the fragmentation of millimeter-size hypervelocity projectiles.
3.2. Impact on Combined Mesh/Plate Bumpers. This section presents results of HVI simulations for the reference configuration with the all-aluminum bumper and the mesh/plate bumper configurations. Figures 7-12 illustrate the evolution of the shapes of debris clouds up to the moment when they reach the control section ( $7 \mathrm{~mm}$ from the bumper), in the case of different bumper configurations and mesh/projectile alignments.

Comparing Figures 7 and 8, one can see that the projectile's impact on the mesh/plate bumper, in the case when the projectile is aligned with a crossover point, leads to the "branching" of the debris cloud with additional "branches" inclined to the initial direction of impact. This effect itself may be considered positive, resulting in better distribution of momentum over the area of the component behind the bumper. However, the size of the leading fragment in Figure $8(\mathrm{c})$, mainly consisting of material of the aluminum plate, 


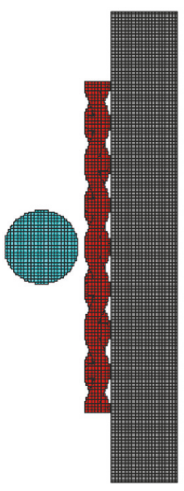

(a)

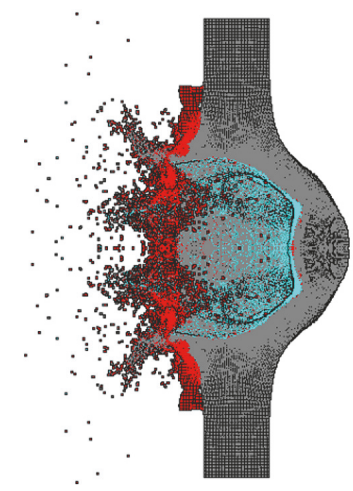

(b)

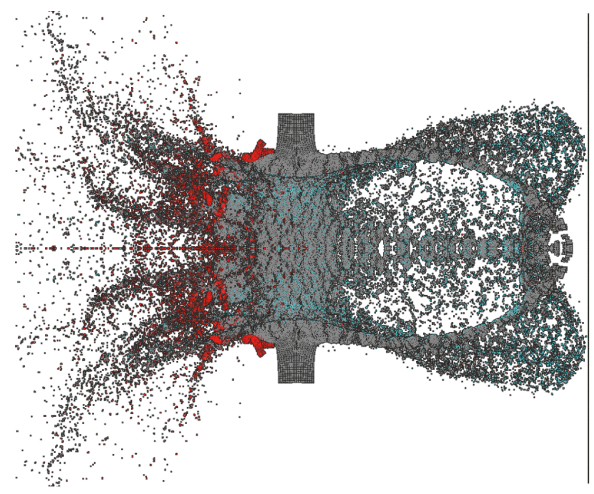

(c)

FIGURE 8: Impact of an aluminum projectile on a combined aluminum mesh-aluminum plate bumper (projectile aligned with crossover point): (a) $t=0$; (b) $t=0.59 \mu \mathrm{s}$; (c) $t=2.57 \mu \mathrm{s}{ }^{*} .{ }^{*}$ Central fragment reaches the control section about $0.13 \mu \mathrm{s}$ later $(\sim 2.7 \mu \mathrm{s})$.

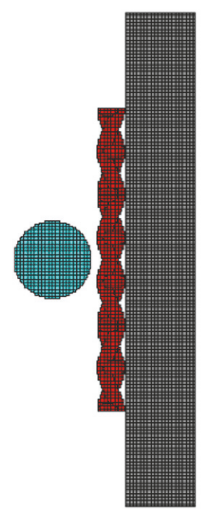

(a)

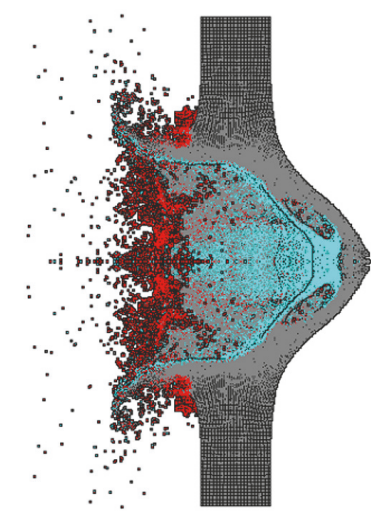

(b)

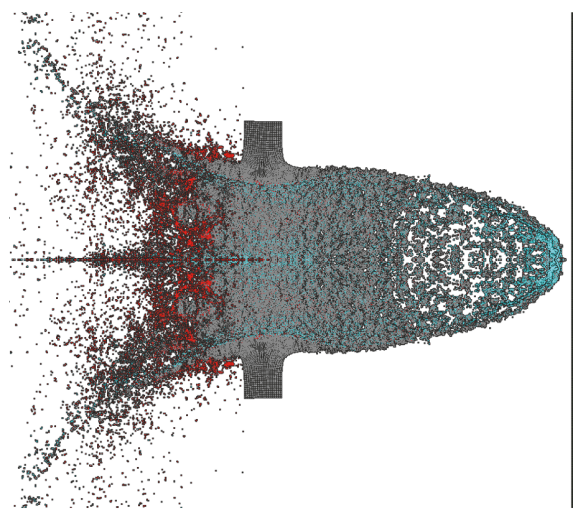

(c)

FIGURE 9: Impact of an aluminum projectile on a combined aluminum mesh-aluminum plate bumper (projectile aligned with aperture): (a) $t=0$; (b) $t=0.55 \mu \mathrm{s}$; (c) $t=2.01 \mu \mathrm{s}$.

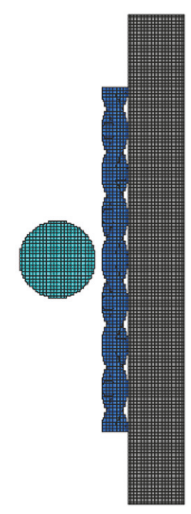

(a)

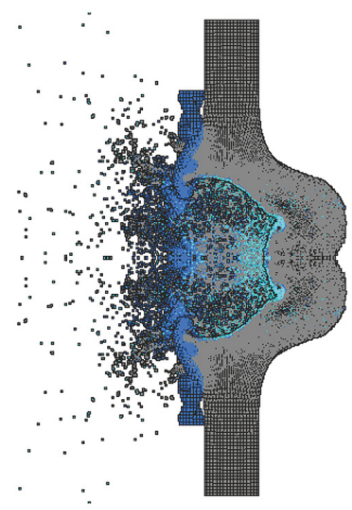

(b)

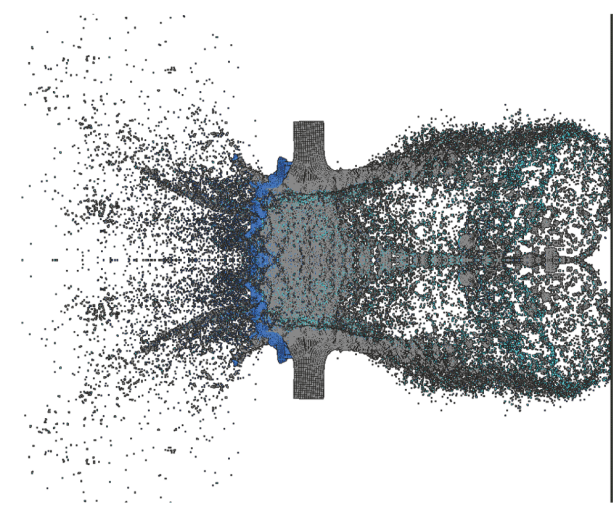

(c)

FIGURE 10: Impact of an aluminum projectile on a combined steel mesh-aluminum plate bumper (projectile aligned with crossover point): (a) $t=0$; (b) $t=0.55 \mu \mathrm{s}$; (c) $t=2.20 \mu \mathrm{s}$. 


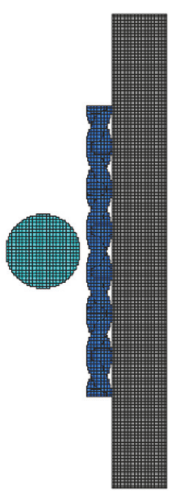

(a)

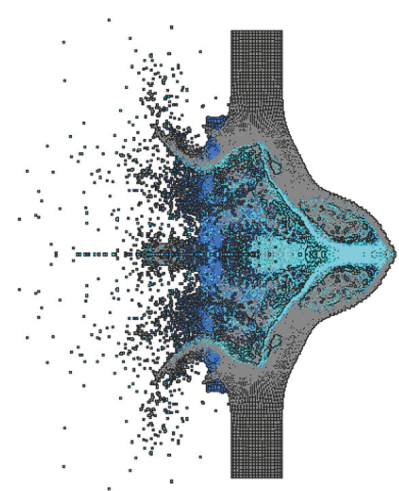

(b)

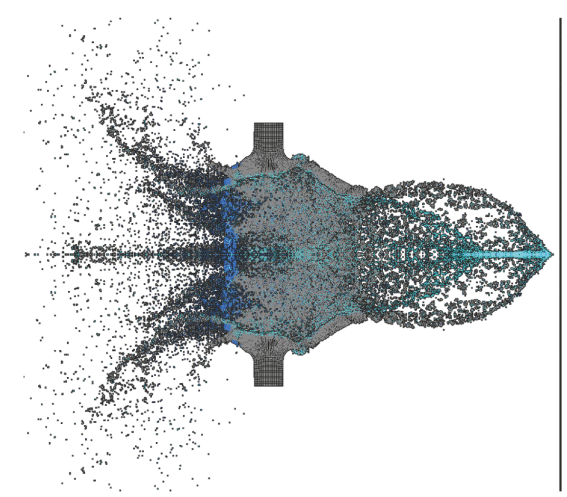

(c)

FIGURE 11: Impact of an aluminum projectile on a combined steel mesh-aluminum plate bumper (projectile aligned with aperture): (a) $t=0$; (b) $t=0.55 \mu \mathrm{s}$; (c) $t=1.65 \mu \mathrm{s}$.

TABLE 3: Parameters of the central fragment.

\begin{tabular}{|c|c|c|c|c|c|c|}
\hline Parameter & Dimensionality & $\mathrm{AL}$ & ALM-1 & ALM-2 & STM-1 & STM-2 \\
\hline Characteristic dimension & $\mathrm{mm}$ & 7.3 & 5.41 & 3.43 & 1.16 & 3.82 \\
\hline Volume & $\mathrm{mm}^{3}$ & 1.0370 & 0.4673 & 0.2223 & 0.0573 & 0.1646 \\
\hline Mass & $\mathrm{mg}$ & 2.69 & 1.23 & 0.575 & 0.153 & 0.421 \\
\hline Average speed & $\mathrm{m} / \mathrm{s}$ & 2635 & 2415 & 3803 & 2752 & 4154 \\
\hline
\end{tabular}

is considerably larger. Further inspection and comparison of Figures 12(a) and 12(b) show that most of the material, in the case of the reference configuration, is distributed over the debris cloud's surface. In contrast, there is a significant concentration of large fragments close to the axis of the debris cloud in the case of the combined mesh/plate bumper.

The projectile's alignment with the center of the aperture (Figure 9) leads to the formation of a relatively large central fragment mainly consisting of the projectile material (see Figure 9(b)). In this case, expansion of the debris cloud is noticeably lower than in the case of impact on the reference configuration (compare Figure 9(c) with Figure 7(c)), which results in a wedge-like shape of the cloud. The central fragment's velocity is quite high, as it reaches the control section at $t \approx 2.00 \mu \mathrm{s}$ (about 9\% earlier than in the case of reference configuration). The aforementioned restraint of the cloud expansion seems to be imposed by the initial "squeezing" of the projectile through the aperture of the mesh.

Use of steel mesh and crossover point alignment of the projectile leads to the large expansion of the debris cloud by the moment it reaches the control section (see Figure 10(c)). Analogous to the case of an aluminum mesh-combined bumper, such impact conditions produce a concentration of a significant amount of material close to the cloud's axis (see Figure 12(d)). This material mainly originates from the aluminum plate of the mesh/plate bumper.

For a steel mesh and projectile aligned with the center of the aperture, the debris cloud's shape is different from the one observed in analogous conditions for an aluminum meshcombined bumper (compare Figure 9(c) and Figure 11(c)).
Another difference is the concentration of projectile material along the axis of the debris cloud (see Figures 11(c) and 12(e)), instead of its spreading over the front surface of the cloud (as in Figure 12(c)). It can be explained as a result of the interaction of the projectile with the steel mesh, which is much denser than projectile material. As a result of the low thickness of the aluminum plate (see Table 1), this configuration provides the lowest deceleration of the debris cloud, which reaches the control section at $t=1.65 \mu \mathrm{s}(25 \%$ earlier than in the case of the reference configuration).

Typically, the central fragments of debris clouds contribute to the damage of spacecraft components located behind them. Parameters of the largest central fragment for each shielding configuration just before reaching the control section were obtained using the "Output Fragment Analysis" function of AUTODYN and are summarized in Table 3. Here "Characteristic dimension" $\left(L_{\mathrm{char}}\right)$ represents the length of the diagonal of the bounding box that contains the fragment completely. It should be noted that because of the smooth distribution of material over the contour of the debris cloud for an all-aluminum bumper (see Figures 7(c) and 12(a)), a large fraction of the debris cloud is considered by AUTODYN as a single fragment, which, therefore, has a large characteristic dimension and comprises 124,132 SPH particles. Central fragments resulting from impacts on the reference and mesh-plate bumpers are shown in Figure 13.

The damaging potential of the central fragments depends on the spatial distribution of their material and fragments' momentum and/or kinetic energy. To account for the spatial distribution of material, the fragments' energy and momentum were averaged over the control area. The latter is the 


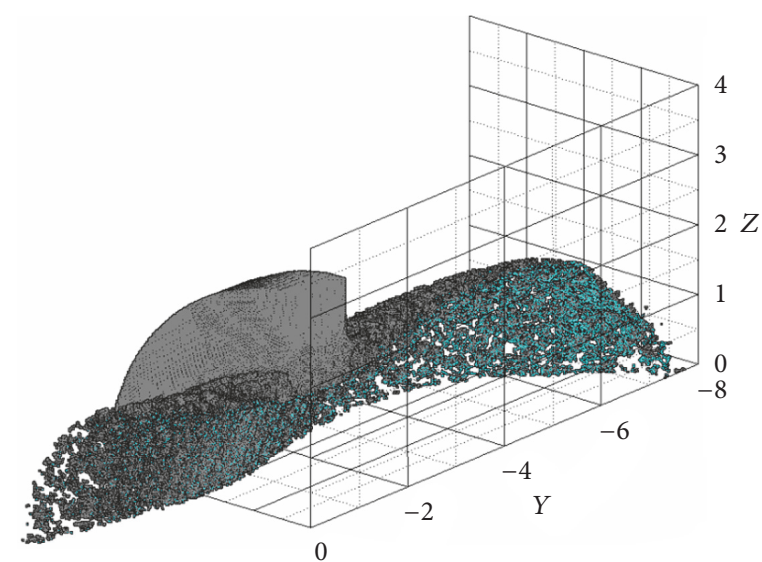

(a)

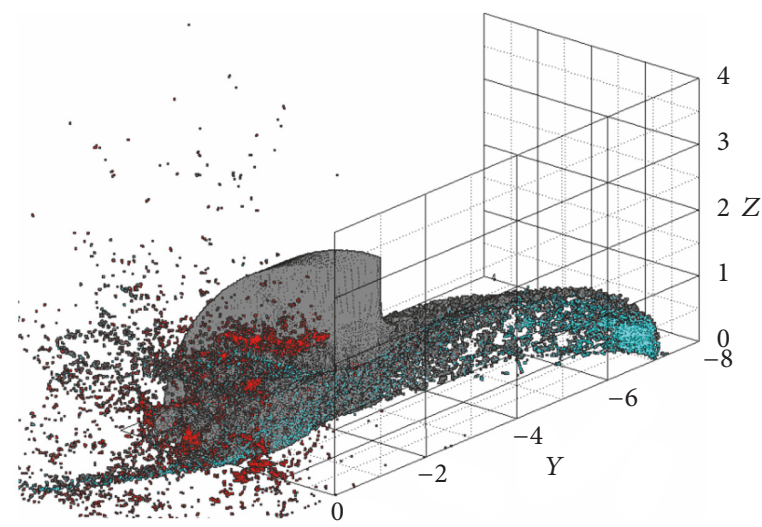

(c)

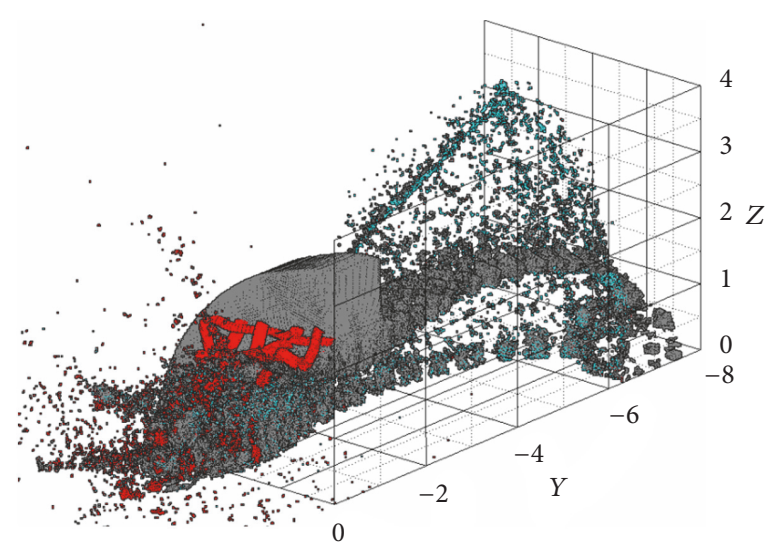

(b)

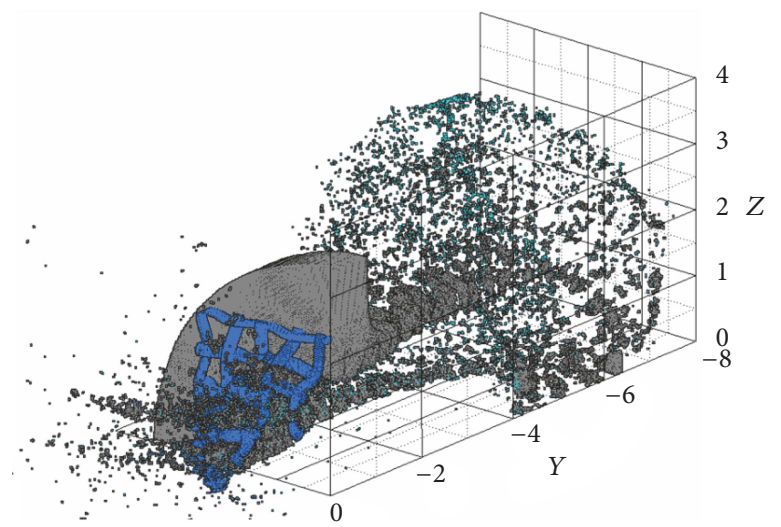

(d)

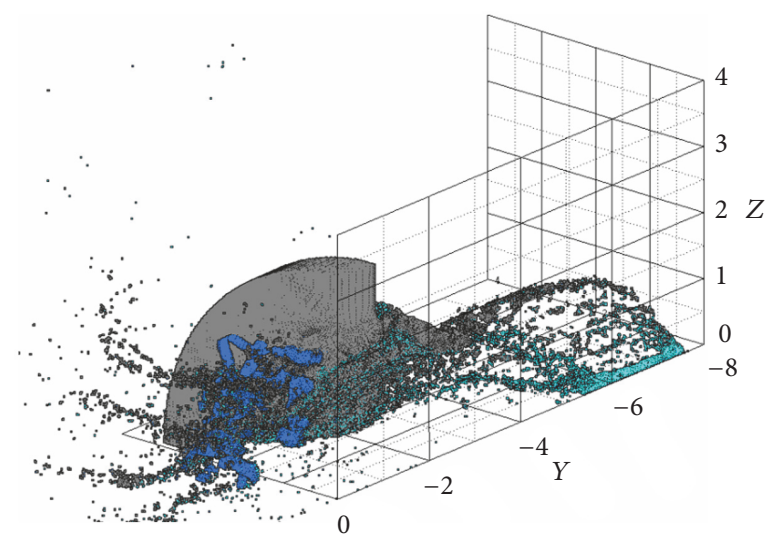

(e)

FIgURE 12: Shapes of the fragment clouds: an isometric view.

area of a circle with diameter equal to the diameter of the fragment's cross-section perpendicular to the impact direction and drawn through the fragment's center of mass, as shown in Figure 13. The damaging potentials of the central fragments in terms of both kinetic energy per unit area and momentum per unit area are summarized in Figure 14 for each shielding/impact configuration.

As it is clearly seen from Figure 14, damaging potentials of the central fragments created as a result of the impact on an aluminum mesh/plate bumper (impact at the center of aperture) and steel mesh/plate bumper (both impact configurations) are much greater than in the case of impact on the all-aluminum bumper. The highest damaging potential is associated with the central fragment formed after impact at the center of the aperture on a steel mesh/plate bumper. This results in its shape elongated in the direction of impact (see Figure 13), which makes this fragment a more efficient penetrator than the other considered fragments that have 

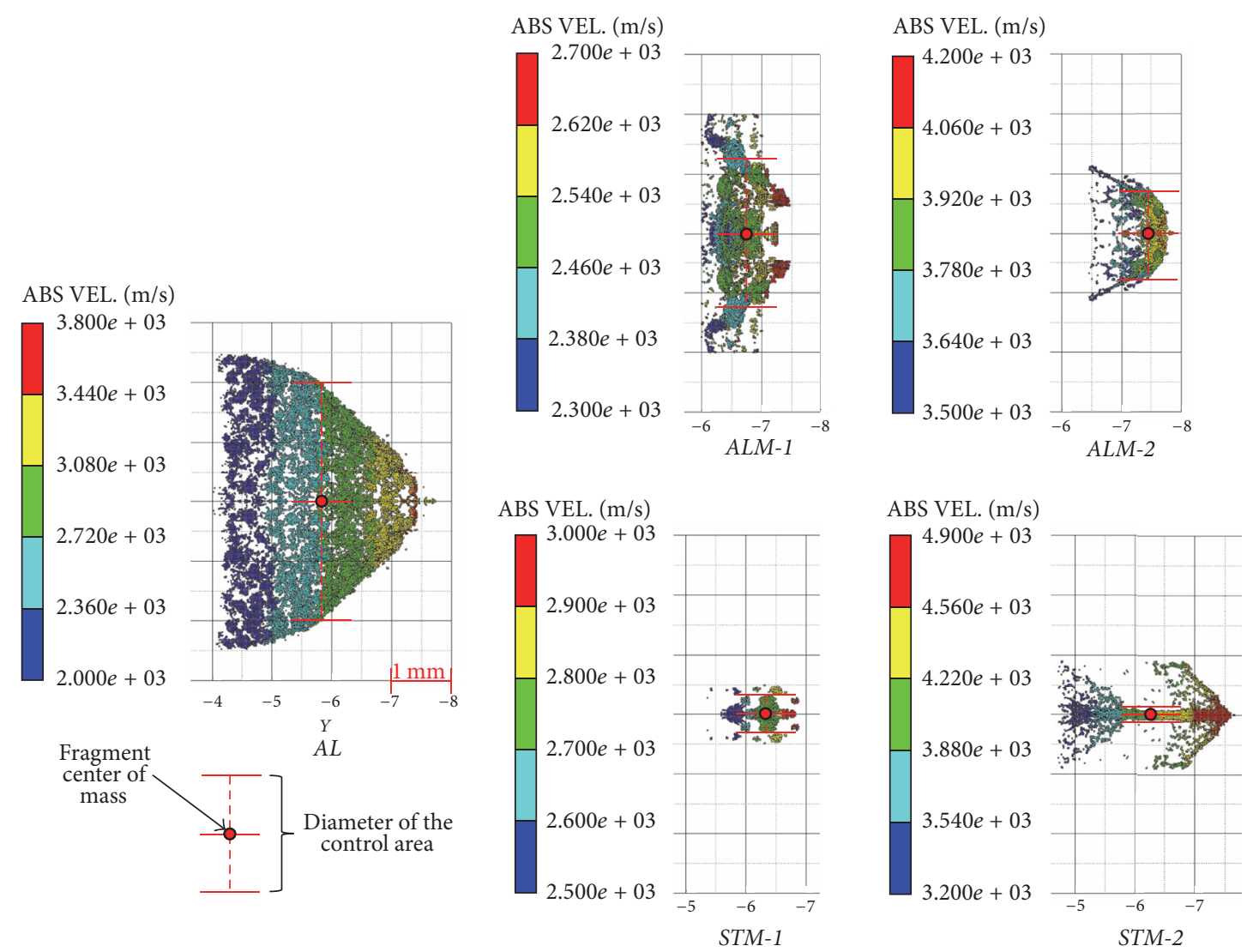

FIGURE 13: Central fragments of debris clouds.

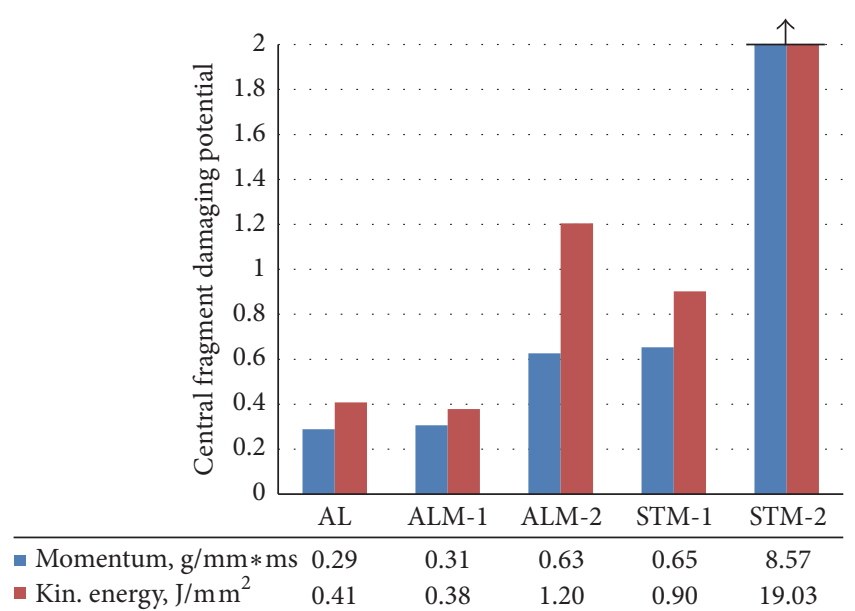

FIGURE 14: The damaging potential of the central fragments.

much larger control areas associated with them. Although crossover-centered impact on a bumper with steel mesh results in lower damaging potential of the central fragment as compared to aperture-centered impact, the fragment's residual momentum and energy are still significantly higher than those that form in case of all-aluminum bumper.

According to Figure 14, central fragments resulting from impacts on all-aluminum bumper and aluminum mesh/plate bumper in the case of projectiles centered at the crossover point have very similar damaging potentials. The energy of the central fragment is even lower for the mesh-plate bumper. However, impact centered with the mesh aperture results in forming a center fragment with a significantly higher damaging potential.

\section{Conclusions}

The following conclusions can be made based on the investigation's results.

First, if the choice is to be made between the steel mesh- and the aluminum mesh-plate bumper, the latter configuration should be preferred, as it demonstrates better efficiency while having the same areal density. This can mainly be attributed to the fact that initial interaction of the projectile with the steel mesh did not provide a strong enough effect to compensate the reduced thickness of the backing aluminum plate.

Second, and most importantly, although combined meshplate bumpers may have some theoretical advantages, the results obtained in this study indicate that no significant increase in the ballistic performance should be expected over a standard aluminum plate bumper. Therefore, the positive results reported in the literature for bumpers with metallic meshes and centimeter-size projectiles do not seem to be scalable down to millimeter-size particles. However, as only one 
combination of dimensional mesh/projectile parameters has been considered in this study, the results do not exclude the possibility of other combinations or impact conditions when fragmentation of small projectiles on combined mesh/plate bumpers will be more efficient.

For the future studies, considering the mesh-plate bumpers with two layers of the aluminum mesh is suggested. These mesh layers should be superposed so the center of the aperture in the first layer of mesh would be at the same relative position as the crossover point in the second layer. This combination might improve the initial fragmentation of a projectile and prevent the formation of a large central fragment as was observed in this study in the case of impacts aligned with the center of the aperture.

\section{Conflicts of Interest}

The authors declare that there are no conflicts of interest regarding the publication of this paper.

\section{Acknowledgments}

This work was supported by Magellan Aerospace Winnipeg, Canadian Space Agency (CSA), and Natural Sciences and Engineering Research Council of Canada (Discovery Grant no. 402115-2012).

\section{References}

[1] A. Cherniaev and I. Telichev, "Weight-efficiency of conventional shielding systems in protecting unmanned spacecraft from orbital debris," Journal of Spacecraft and Rockets, vol. 54, no. 1, pp. 75-89, 2017.

[2] E. L. Christiansen, J. Arnold, A. Davis et al., "Handbook for designing MMOD protection," NASA/TM-2009-214785, NASA Johnson Space Center, Houston, Tex, USA, 2009.

[3] E. L. Christiansen, K. Nagy, D. M. Lear, and T. G. Prior, "Space station MMOD shielding," Acta Astronautica, vol. 65, no. 7-8, pp. 921-929, 2009.

[4] R. Destefanis, F. Schäfer, M. Lambert, M. Faraud, and E. Schneider, "Enhanced space debris shields for manned spacecraft," International Journal of Impact Engineering, vol. 29, no. 1-10, pp. 215-226, 2003.

[5] M. Higashide, M. Tanaka, Y. Akahoshi, S. Harada, and F. Tohyama, "Hypervelocity impact tests against metallic meshes," International Journal of Impact Engineering, vol. 33, no. 1-12, pp. 335-342, 2006.

[6] N. N. Myagkov, T. A. Shumikhin, and L. N. Bezrukov, "Experimental and numerical study of peculiarities at high-velocity interaction between a projectile and discrete bumpers," International Journal of Impact Engineering, vol. 37, no. 9, pp. 980-994, 2010.

[7] E. L. Christiansen and J. H. Kerr, "Mesh double-bumper shield: a low-weight alternative for spacecraft meteoroid and orbital debris protection," International Journal of Impact Engineering, vol. 14, no. 1-4, pp. 169-180, 1993.

[8] F. Horz, M. Cintala, T. See et al., "Comparison of continuous and discontinuous collisional bumpers: dimensionally scaled impact experiments into single wire meshes," Tech. Rep. 104749, NASA, Washington, DC, USA, 1992.
[9] ANSYS AUTODYN User's Manual. Release 15.0, 2013.

[10] J. J. Monaghan, "Smoothed particle hydrodynamics," Annual Review of Astronomy and Astrophysics, vol. 30, no. 1, pp. 543574, 1992.

[11] C. J. Hayhurst, I. H. Livingstone, R. Clegg, G. E. Fairlie, S. J. Hiermaier, and M. Lambert, "Numerical simulation of hypervelocity impacts on aluminum and Nextel/Kevlar Whipple shields," in Proceedings of the Hypervelocity Shielding Workshop, Galveston, Tex, USA, March 1998.

[12] S. K. Roy, M. Trabia, B. O’Toole et al., "Study of hypervelocity projectile impact on thick metal plates," Shock and Vibration, vol. 2016, Article ID 4313480, 11 pages, 2016.

[13] D. Steinberg, Equation of State and Strength Properties of Selected Materials, Lawrence Livermore National Laboratory, Livermore, Calif, USA, 1996.

[14] M. A. Meyers, Dynamic Behavior of Materials, John Wiley \& Sons, Inc., Hoboken, NJ, USA, 1994.

[15] G. R. Johnson and W. H. Cook, "Fracture characteristics of three metals subjected to various strains, strain rates, temperatures and pressures," Engineering Fracture Mechanics, vol. 21, no. 1, pp. 31-48, 1985.

[16] B. M. Corbett, Hypervelocity impact damage response and characterization of thin plate targets at elevated temperatures [Ph.D. thesis], University of Denver, Denver, Colo, USA, 2008.

[17] N. K. Gupta, M. A. Iqbal, and G. S. Sekhon, "Experimental and numerical studies on the behavior of thin aluminum plates subjected to impact by blunt- and hemispherical-nosed projectiles," International Journal of Impact Engineering, vol. 32, no. 12, pp. 1921-1944, 2006. 

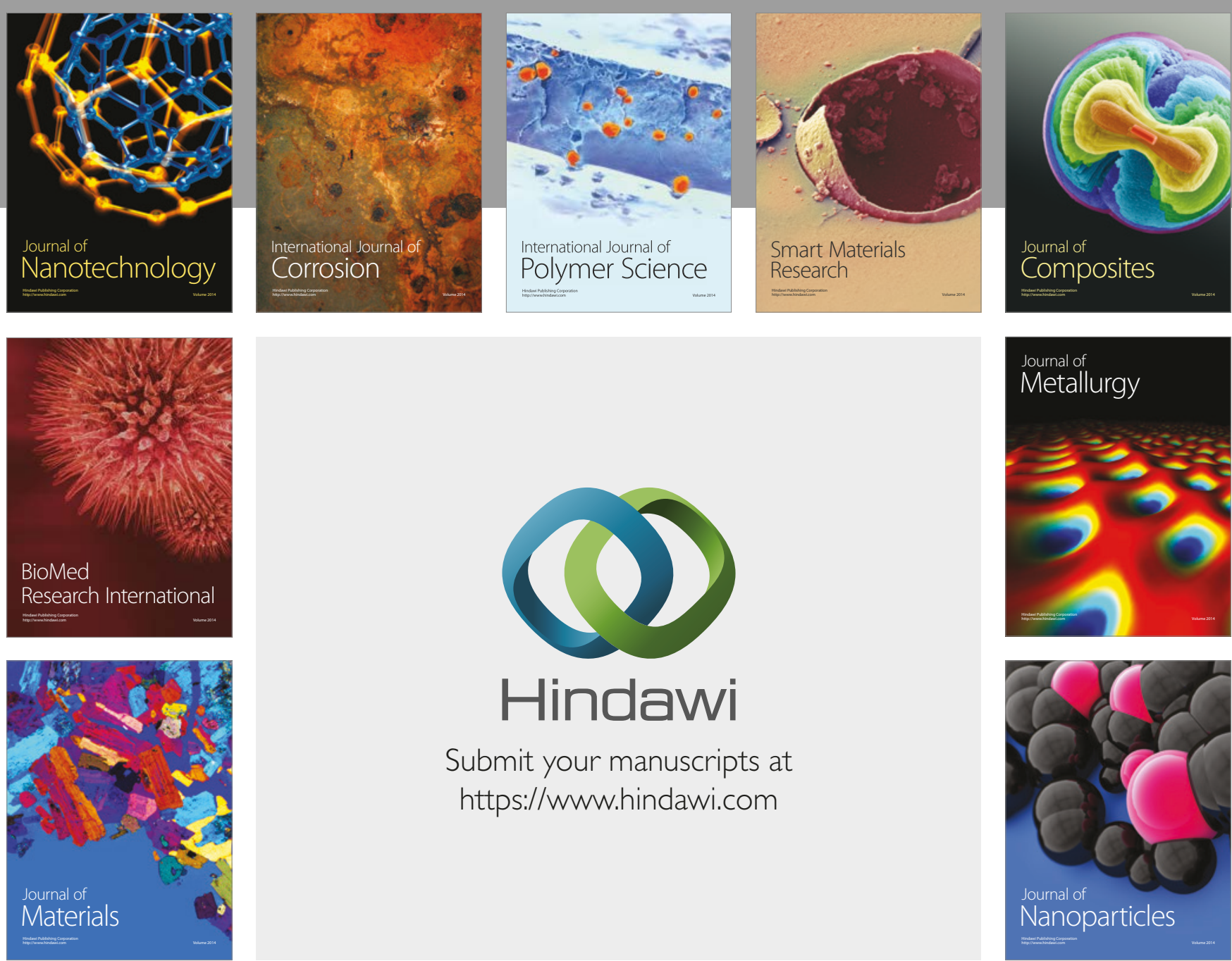

\section{Hindawi}

Submit your manuscripts at

https://www.hindawi.com
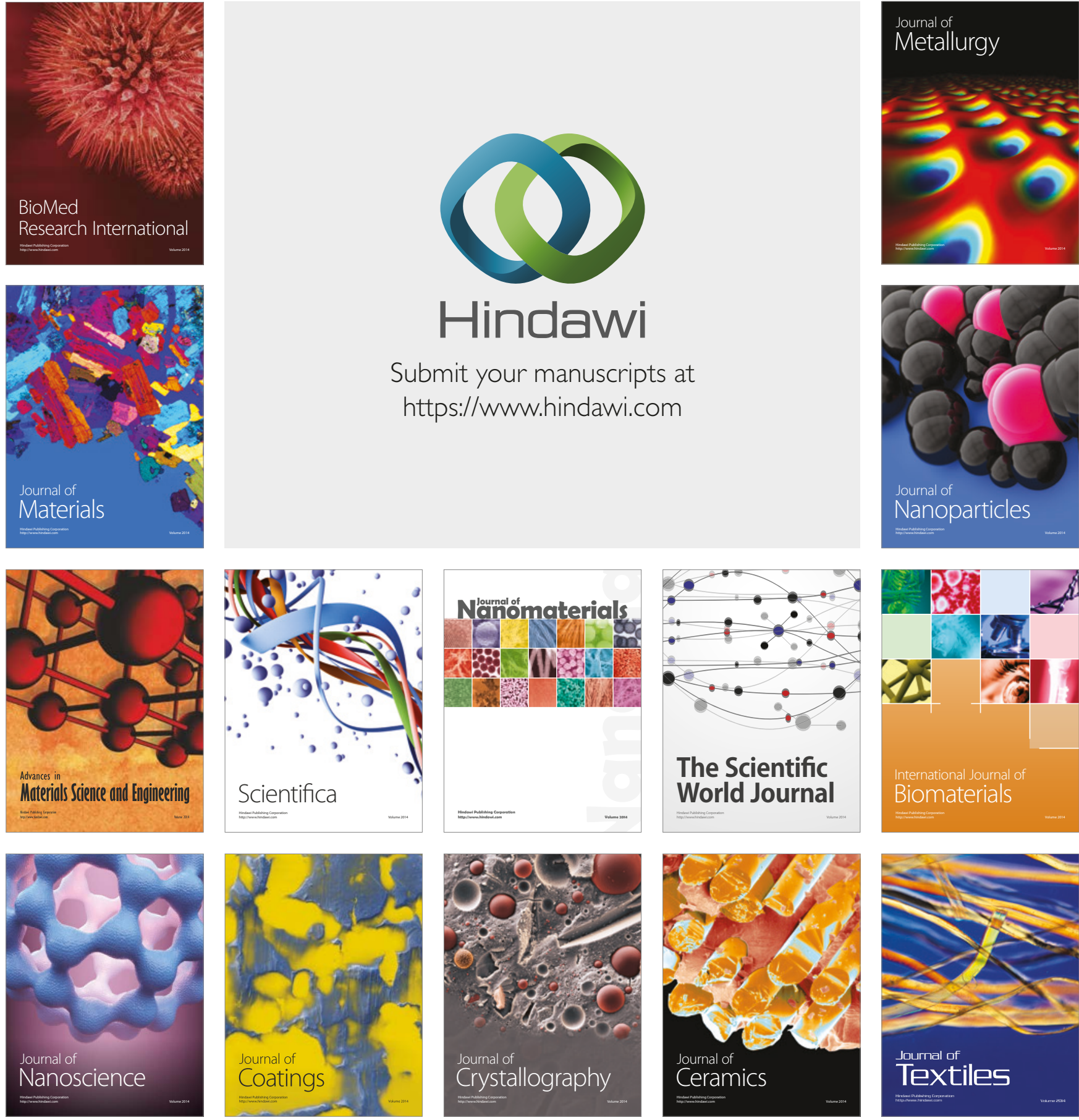

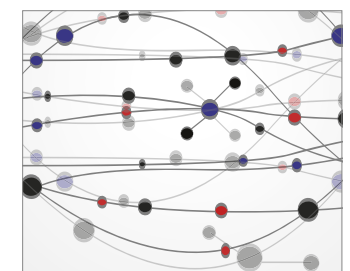

The Scientific World Journal
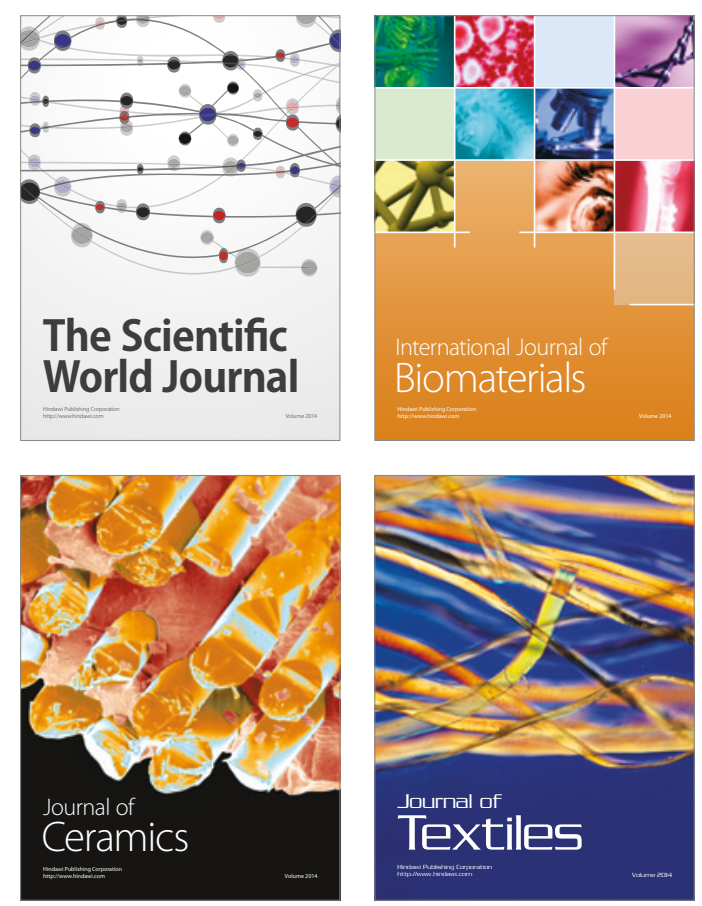